\title{
Coordination Model of Consumer Information Sharing in the Online Tourism Supply Chain
}

\author{
Honglian Guo, Xuexue Zhang* \\ Beijing Wuzi University, Beijing 101149, China \\ *Corresponding author: Xuexue Zhang, zhangxuexue0709@163.com
}

\begin{abstract}
This is a study of the coordination model of consumer information sharing in the tourism supply chain with ecommerce platforms as the core. On the basis of considering the risks, a game theory is used to explore the information sharing effort level and the output profit of the tourism supply chain under both, centralized decision-making and decentralized decision-making. Finally, numerical simulation is used to verify the model and put forward a method of coordination for all parties in the tourism supply chain to achieve maximum profit.
\end{abstract}

Keywords: Tourism supply chain; Information sharing; Coordination

Publication date: December 2021; Online publication: December 31, 2021

\section{Introduction}

In recent years, the scale of online tourism transactions in China has reached $40 \%$. Even with the COVID19 pandemic, the online tourism market still reached RMB 2.03 trillion in 2020, a 3.4\% increase from the previous year. However, the rapid development of online tourism has brought about new challenges. The online consumer dispute mediation platform, "Dian Su Bao," showed that online tourism is facing several problems, such as false propaganda, difficult refunds, and increase in prices. Guangming Net pointed out that some individual enterprises have low quality management level and non-standardized operations, thus infringing on the legitimate rights and interests of tourists as well as disrupting the order of the tourism market. This is because the profit demand of e-commerce platform leads to its control of input cost and concealment of collected consumer information, and some tourism suppliers blindly pursue immediate interests. There is a lack of communication when the two: the problem of information sharing has not been solved, and consumers' individual needs and service quality cannot be guaranteed. Solving this problem can give full play to the unique information exchange and sharing function of online tourism platforms, maximize the benefits, and promote the sustainable development of tourism. Therefore, online tourism supply chain has attracted great attention from enterprises and scholars.

From the meaning of "tourism supply chain," it is different from the traditional service supply chain. Huang Meng proposed that the online tourism supply chain mainly comprises of internet enterprises with e-commerce platforms as the core, and travel agencies, scenic spots, hotels, etc. as tourism suppliers ${ }^{[1]}$. Zheng Siwei talked about the tourism supply chain as a supply chain system that includes all tourism services and products to meet the needs of consumers through online search, reservation, and transaction sharing ${ }^{[2]}$. Mohamed studied the operation mode of online travel agencies and found that most travel agencies use online platforms to promote their travel services and products; he also noted that through this, the revenue of travel agencies increased significantly ${ }^{[3]}$. Daugherty had pointed out that tickets for scenic spots, online hotel reservations, and consumption have brought tourists richer travel experience ${ }^{[4]}$. Romero proposed that in e-commerce, new media will serve as the necessary bridge for communication between 
tourism suppliers and consumer ${ }^{[5]}$. Ji Yanan had suggested that e-commerce platforms must allocate profits and increase the market demand on the premise of ensuring its rapid speed of development ${ }^{[6]}$. From the analysis of the characteristics of the online tourism supply chain, there are relatively many constituent elements of e-commerce platforms, and the links of the elements are dynamic ${ }^{[7]}$. Danuta pointed out that the product development of the tourism supply chain has its particularity, which needs to be continuously updated to meet the expectations of tourism consumers ${ }^{[8]}$. Huang believes that the solution to improve the overall revenue of the tourism supply chain is to build an overall revenue model ${ }^{[9]}$. $\mathrm{Pu} \mathrm{Xu}$ Jin and others have constructed a game model and obtained the pricing as well as the sales effort ${ }^{[10]}$. Yong Long pointed out that service costs, commissions, and compensation rates have an impact on the adopted strategies ${ }^{[11]}$.

Considering the above development and background of the tourism supply chain, this research studies the information sharing of the tourism supply chain under different decisions and constructs a coordination model of information sharing.

\section{Model description and assumptions}

Considering a secondary supply chain consisting of an e-commerce platform with multiple tourism suppliers, the assumptions are as follows:

(1) the e-commerce platform is the core enterprise, while the tourism suppliers are the cooperative enterprises; the supplier enterprise is set $S=\left\{s_{1}, s_{2}, s_{3} \ldots s_{n}\right\}$;

(2) the e-commerce platform is risk-neutral, only the risk attitudes of the tourism suppliers are considered; the risk cost is $\frac{\rho_{i} \operatorname{Var}\left(\pi_{i}\right) \sigma^{2}}{2}$ and the risk factor is $\rho_{i}$;

(3) the e-commerce platform's sharing effort level is $e_{1}$, and each supplier's information sharing effort level is $e_{i} \in\left(0, e_{i}\right), e_{i}(i=1,2,3 \ldots, n)$ represents the $i$-th travel supplier.

(4) the cost of information sharing is $C=\frac{c e^{2}}{2}$, where $c$ is the cost coefficient;

(5) the output benefit of information sharing, $R\left(e_{1}, e_{i}\right)=g_{1} \ln e_{1}+\sum_{i=1}^{n} g_{i} \ln e_{i}+\eta$, where $\eta$ is a random variable, which obeys the normal distribution $N \sim\left(0, \sigma^{2}\right)$;

(6) the profit ratio shared by the e-commerce platform is $\beta_{i}$, and the fixed expenses for the suppliers' purchase information is $\alpha_{i}$;

(7) the retention benefit of the tourism supplier is divided into $\bar{w}$.

\section{Centralized decision-making}

In centralized decision-making, the two sides make joint decisions. The e-commerce platform can observe the effort of the suppliers. At this time, the profit is not affected by random error, and the risk factor for the tourism suppliers, $\rho_{i}=0$.

$$
\begin{aligned}
& \max \pi=g_{1} \ln e_{1}+\sum_{i=1}^{n}\left(g_{i} \ln e_{i}-\frac{c_{i}}{2} e_{i}^{2}\right)+\eta-\frac{c_{1}}{2} e_{1}^{2}-\frac{\rho_{i}\left(1-\beta_{i}\right)^{2} \sigma^{2}}{2} \\
& \text { Let } \frac{\partial \max \pi}{\partial e_{1}}=g_{1} \frac{1}{e_{1}}-c_{1} e_{1}=0 \quad e_{1}=\sqrt{\frac{g_{1}}{c_{1}}}, \quad e_{i}=\sqrt{\frac{g_{i}}{c_{i}}}
\end{aligned}
$$


The profits of the two parties are as follows:

$$
\begin{aligned}
& \pi_{1}=\sum_{i=1}^{n} \beta_{i}\left(\frac{n g_{1}}{2} \ln \frac{g_{1}}{c_{1}}+\frac{g_{i}}{2} \ln \frac{g_{i}}{c_{i}}+\eta\right)+\sum_{i=1}^{n} \alpha_{i}-\frac{c_{1}}{2} e_{1}^{2} \\
& \pi_{i}=\left(1-\beta_{i}\right)\left(\frac{g_{1}}{2} \ln \frac{g_{1}}{c_{1}}+\frac{g_{i}}{2} \ln \frac{g_{i}}{c_{i}}+\eta\right)-\alpha_{i}-\frac{c_{i}}{2} e_{i}^{2}
\end{aligned}
$$

The total profit of the supply chain is as follows:

$$
\pi=\frac{g_{1}}{2} \ln \frac{n g_{1}}{c_{1}}+\sum_{i=1}^{n} \frac{g_{i}}{2} \ln \frac{g_{i}}{c_{i}}+\eta-\frac{n g_{1}}{2}-\sum_{i=1}^{n} \frac{g_{i}}{2}
$$

\section{Decentralized decision-making}

\subsection{Dynamic game}

In the tourism supply chain, e-commerce platforms connect consumers and suppliers, thus enjoying more private information. Therefore, the online tourism supply chain is a stackelberg game dominated by ecommerce platforms. The r-commerce platform first decides its strategy $\left\{\alpha_{i}, \beta_{i}\right\}$. Then, the supplier decides the $e_{i}$. Using backward induction to solve:

$$
\begin{aligned}
& \max \pi_{i}=(1-\beta)\left(g_{1} \ln e_{1}+g_{i} \ln e_{i}+\eta\right)-\alpha_{i}-\frac{c_{i}}{2} e_{i}^{2}-\frac{\rho_{i}\left(1-\beta_{i}\right)^{2} \sigma^{2}}{2} \\
& \text { Let } \frac{\partial \pi_{i}}{\partial e_{i}}=\left(1-\beta_{i}\right) \frac{g_{i}}{e_{i}}-c_{i} e_{i}=0 \quad \text { get } \quad e_{i}=\sqrt{\frac{g_{i}\left(1-\beta_{i}\right)}{c_{i}}}
\end{aligned}
$$

Substituting the formula (6) into $\pi_{1}$ :

$$
\begin{aligned}
& \max \pi_{1}=\sum_{i=1}^{n} \beta_{i}\left(g_{1} \ln e_{1}+g_{i} \ln \sqrt{\frac{g_{i}\left(1-\beta_{i}\right)}{c_{i}}}+\eta\right)+\sum_{i=1}^{n} \alpha_{i}-\frac{c_{1}}{2} e_{1}^{2} \\
& \text { Let } \frac{\partial \pi_{1}}{\partial e_{1}}=\beta_{i} \frac{g_{1}}{e_{1}}-c_{1} e_{1}=0, \text { get } \quad e_{1}=\sqrt{\frac{n g_{1} \beta_{i}}{c_{1}}} \\
& \frac{\partial \pi_{1}}{\partial \beta_{i}}=\mathrm{g}_{1} \ln e_{1}+\frac{g_{i}}{2} \ln \frac{g_{i}\left(1-\beta_{i}\right)}{c_{i}}+\eta-\frac{\beta_{i} g_{i}}{2\left(1-\beta_{i}\right)}=0
\end{aligned}
$$

Combining (9) and (10):

$$
\frac{\mathrm{g}_{1}}{2} \ln \frac{n g_{1} \beta_{i}}{c_{1}}+\frac{g_{i}}{2} \ln \frac{g_{i}\left(1-\beta_{i}\right)}{c_{i}}+\eta-\frac{\beta_{i} g_{i}}{2\left(1-\beta_{i}\right)}=0 \quad, \quad \alpha_{i}=\frac{\beta_{i} g_{i}}{2}-\frac{g_{i}\left(1-\beta_{i}\right)}{2}-\frac{\rho_{i}\left(1-\beta_{i}\right)^{2} \sigma^{2}}{2}
$$


Maximize the profit when the strategy developed by the e-commerce platform meets the above relationship. $\frac{\partial e_{i}}{\partial \beta_{i}}=-\frac{g_{i}}{2} \sqrt{\frac{g_{i}}{c_{i}\left(1-\beta_{i}\right)}} \leq 0$; namely, the higher the information sharing level of the tourism supplier, the larger the share of information sharing revenue. The profits of both parties are as follows:

$$
\begin{aligned}
& \pi_{1}^{*}=\sum_{i=1}^{n}\left(\frac{g_{1}}{2} \ln \frac{n g_{1} \beta_{i}}{c_{1}}+\frac{g_{i}}{2} \ln \frac{g_{i}\left(1-\beta_{i}\right)}{c_{i}}+\alpha_{i}-\frac{g_{1} \beta_{i}}{2}\right)+\eta \\
& \pi_{i}^{*}=\frac{\mathrm{g}_{1}}{2} \ln \frac{n g_{1} \beta_{i}}{c_{1}}+\frac{g_{i}}{2} \ln \frac{g_{i}\left(1-\beta_{i}\right)}{c_{i}}+\eta-\alpha_{i}-\frac{g_{i}\left(1-\beta_{i}\right)}{2}-\frac{\rho_{i}\left(1-\beta_{i}\right)^{2} \sigma^{2}}{2}
\end{aligned}
$$

The overall profit of the supply chain is as follows:

$$
\pi^{*}=\sum_{i=1}^{n}\left[\frac{g_{1}}{2} \ln \frac{n g_{1} \beta_{i}}{c_{1}}+\frac{g_{i}}{2} \ln \frac{g_{i}\left(1-\beta_{i}\right)}{c_{i}}-\frac{g_{i}\left(1-\beta_{i}\right)}{2}-\frac{g_{1} \beta_{i}}{2}-\frac{\rho_{i}\left(1-\beta_{i}\right)^{2} \sigma^{2}}{2}\right]+\eta
$$

\subsection{Static game}

In this case, both parties make decisions simultaneously. The efforts of both e-commerce platforms and suppliers in the tourism supply chain affect the output. E-commerce platforms are unable to observe the degree of information sharing efforts of tourism suppliers, so the output is also affected by random variables.

$$
\begin{aligned}
& \max \pi_{1}=\sum_{i=1}^{n} \beta_{i}\left(g_{1} \ln e_{1}+g_{i} \ln e_{i}+\eta\right)+\sum_{i=1}^{n} \alpha_{i}-\frac{c_{1}}{2} e_{1}^{2} \\
& \text { s.t. } \pi_{i} \geq \bar{w} \quad(I R) \\
& e_{i} \in \max \pi_{i} \quad \text { (IC) }
\end{aligned}
$$

Constraint $(I R)$ is the supplier participation constraint; the revenue obtained after information sharing is greater than the retention utility. Constraint $(I C)$ is the incentive compatibility constraint of the supplier; the tourism supplier makes the best effort to participate in the decision-making. A Lagrangian function is constructed as follows:

$$
\begin{aligned}
& L=\sum_{i=1}^{n} \beta_{i}\left(g_{1} \ln e_{1}+g_{i} \ln e_{i}+\eta\right)+\sum_{i=1}^{n} \alpha_{i}-\frac{c_{1}}{2} e_{1}^{2}+\lambda_{1}\left[\left(1-\beta_{i}\right) \frac{g_{i}}{e_{i}}-c_{i} e_{i}\right] \\
& \lambda_{2}\left[\left(1-\beta_{i}\right)\left(g_{1} \ln e_{1}+g_{i} \ln e_{i}+\eta\right)-\alpha_{i}-\frac{c_{i}}{2} e_{i}^{2}-\frac{\rho\left(1-\beta_{i}\right)^{2} \sigma^{2}}{2}\right]
\end{aligned}
$$

Solving the above formula, let

$$
\frac{\partial L}{\partial \lambda_{1}}=\left(1-\beta_{i}\right) \frac{g_{i}}{e_{i}}-c_{i} e_{i}=0
$$




$$
\begin{aligned}
& \frac{\partial L}{\partial \lambda_{2}}=\left(1-\beta_{i}\right)\left(g_{1} \ln e_{1}+g_{i} \ln e_{i}+\eta\right)-\alpha_{i}-\frac{c_{i}}{2} e_{i}^{2}-\frac{\rho_{i}\left(1-\beta_{i}\right)^{2} \sigma^{2}}{2}-\bar{w}=0 \\
& \frac{\partial L}{\partial e_{1}}=\sum_{i=1}^{n} \beta_{i} \frac{g_{1}}{e_{1}}-c_{1} e_{1}+\lambda_{2}\left(1-\beta_{i}\right) \frac{g_{1}}{e_{1}}=0 \\
& \frac{\partial L}{\partial e_{i}}=\beta_{i} \frac{g_{i}}{e_{i}}+\lambda_{1}\left[(1-\beta) \frac{g_{i}}{e_{i}}-c_{i} e_{i}\right]+\lambda_{2}\left[\left(\beta_{i}-1\right) \frac{g_{i}}{e_{i}}-c_{i}\right]=0 \\
& \frac{\partial L}{\partial \alpha_{i}}=n-\lambda_{2}=0 \\
& \frac{\partial L}{\partial \beta_{i}}=n\left(g_{1} \ln e_{1}+g_{i} \ln e_{i}+\eta\right)-\lambda_{1} \frac{g_{2}}{e_{2}}+\lambda_{2}\left[-\left(g_{1} \ln e_{1}+g_{i} \ln e_{i}+\eta\right)+\rho_{i}\left(1-\beta_{i}\right) \sigma^{2}\right]=0 \\
& \rho \neq 0, \text { obtain the solution } \\
& e_{1}=\sqrt{\frac{g_{1}}{c_{1}}}, e_{i}=\sqrt{\frac{g_{i}\left(1-\beta_{i}\right)}{c_{i}}}, \beta_{i}=1-\frac{g_{i}}{2 \rho_{i} \sigma^{2}}, \quad \alpha_{i}=\left(1-\beta_{i}\right)\left(g_{1} \ln e_{1}+g_{i} \ln e_{i}+\eta\right)-\frac{c_{i}}{2} e_{i}^{2}-\frac{\rho_{i}\left(1-\beta_{i}\right)^{2} \sigma^{2}}{2}-\bar{w}
\end{aligned}
$$

From these formulas, when the parameter satisfies $0<g_{i}<2 \rho_{i} \sigma^{2}$ and $\frac{g_{i}}{2 \rho_{i} \sigma^{2}}>c_{i}$, the hypothesis is valid. $\frac{\partial e_{1}}{\partial \beta_{i}}>0, \frac{\partial e_{i}}{\partial \beta_{i}}<0$, the harder the supplier works, the less the e-commerce platform will be divided; the harder the e-commerce platform works, the more shares it would have.

$$
\begin{aligned}
& \pi_{1}^{* *}=\sum_{i=1}^{n} \beta_{i}\left(\frac{g_{1}}{2} \ln \frac{n g_{1}}{c_{1}}+\frac{g_{i}}{2} \ln \frac{g_{1}\left(1-\beta_{i}\right)}{c_{i}}\right)+\sum_{i=1}^{n} \alpha_{i}-\frac{g_{1}}{2} \\
& \pi_{i}^{* * *}=\left(1-\beta_{i}\right)\left(\frac{g_{1}}{2} \ln \frac{g_{1}}{c_{1}}+\frac{g_{i}}{2} \ln \frac{g_{i}\left(1-\beta_{i}\right)}{c_{i}}+\eta\right)-\alpha_{i}-\frac{g_{i}\left(1-\beta_{i}\right)}{2}
\end{aligned}
$$

The overall profit of the supply chain is as follows:

$$
\pi^{* *}=\frac{g_{1}}{2} \ln \frac{n g_{1}}{c_{1}}+\sum_{i=1}^{n} \frac{g_{i}}{2} \ln \frac{g_{i}\left(1-\beta_{i}\right)}{c_{i}}+\eta-\frac{n g_{1}}{2}-\sum_{i=1}^{n}\left[\frac{g_{i}\left(1-\beta_{i}\right)}{2}+\frac{\rho_{i}\left(1-\beta_{i}\right)^{2} \sigma^{2}}{2}\right]
$$

\section{Simulation}

\subsection{Numerical simulation}

This study is verified and analyzed with the help of examples, and relevant values are assigned as shown in Table 1. 
Table 1. Parameters

\begin{tabular}{ccccccc}
\hline$g l$ & $g i$ & $C 1$ & $c i$ & $\eta$ & $\rho$ & $\sigma$ \\
\hline 0.5 & 0.7 & 0.1 & 0.5 & 2 & 0.4 & 1 \\
\hline
\end{tabular}

In order to make the result more intuitive, set $n=1$. The two have the highest effort level and the highest profit under centralized decision-making; under the dynamic game, the two have the lowest effort level and the lowest profit, whereas under the static game, the e-commerce platform's effort level is equal to that of the centralized decision-making and the supplier's effort level as well as the profit are higher than those of the dynamic game (Table 2).

Table 2. Numerical simulation under centralized decision-making and decentralized decision-making

\begin{tabular}{cccc}
\hline & Centralized Decision & Dynamic Game & Static Game \\
\hline$e 1$ & 2.24 & 1.07 & 2.24 \\
$e i$ & 1.18 & 1.04 & 1.1 \\
$\beta$ & - & 0.23 & 0.125 \\
$\pi l$ & - & 0.42 & 0.07 \\
$\pi i$ & - & 1.2 & 1.7 \\
$\pi$ & 1.92 & 1.62 & 1.77 \\
\hline
\end{tabular}

\subsection{Sensitivity analysis}

\subsubsection{Centralized decision-making}

In centralized decision-making, the maximization of the overall revenue is considered, and it has nothing to do with the risk. As shown in Figure 1, the greater the effort coefficient, the more profit. The higher the cost factor, the lower the level of information sharing effort and the profit.
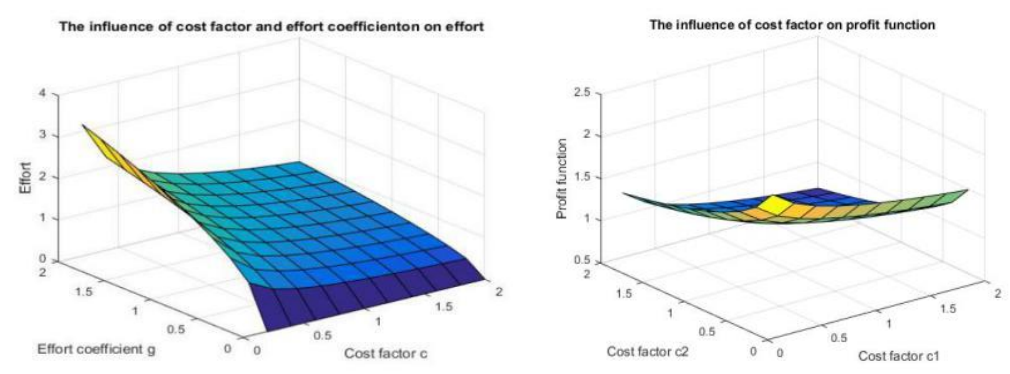

Figure 2.
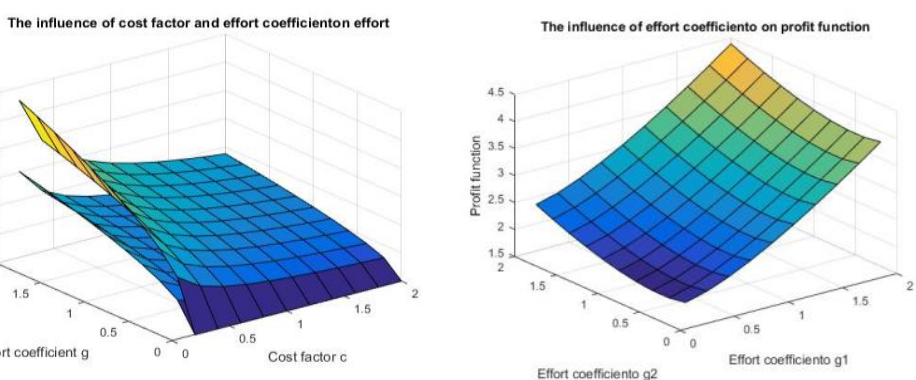

Figure 1. Sensitivity analysis under centralized decision-making. Figure 2. Influence of cost factor and effort coefficient on effort under the dynamic game 


\subsubsection{Sensitivity analysis of dynamic game under decentralized decision-making}

As shown in Figure 2 and Figure 3, the greater the cost coefficient, the lower the effort the two parties make and the profit. The greater the effort coefficient, the more effort the two sides make, and the profit is higher. The party with the larger cost coefficient has less profit share, while the party with the greater effort coefficient has more profit share.
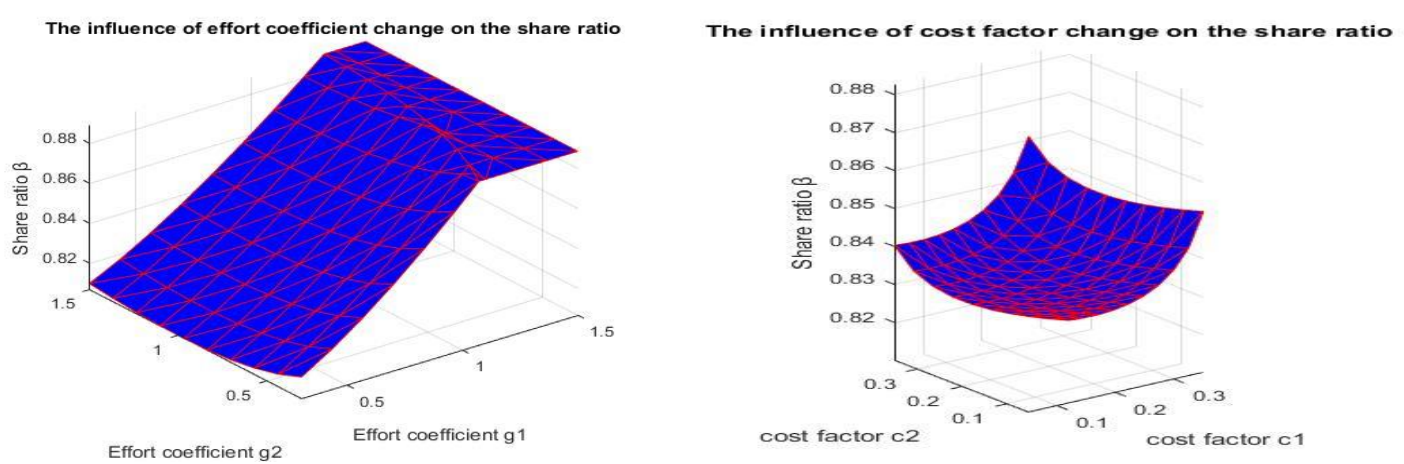

Figure 3. Influence of cost factor and effort coefficient on share ratio

Figure 4 shows that the higher the risk coefficient, the lower the profit. The larger the random influencing factors, the smaller the profit function.
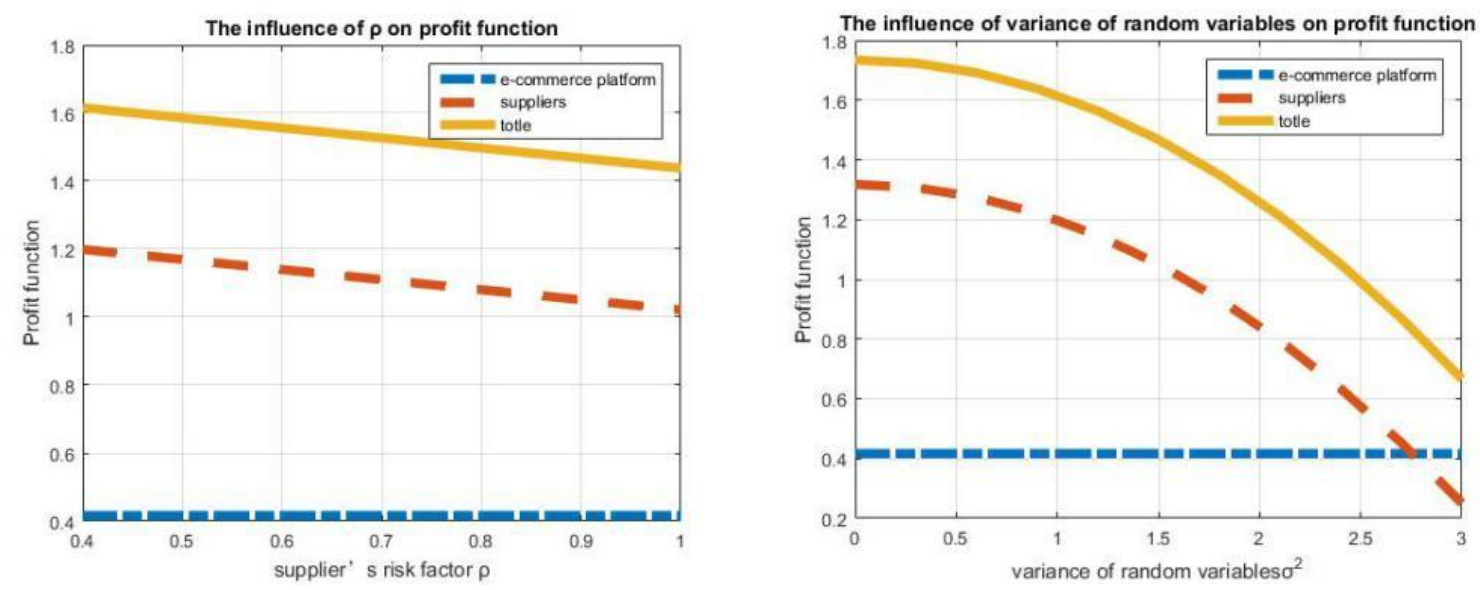

Figure 4. Influence of supplier's risk factor and variance of random variables on profit function

\subsubsection{Sensitivity analysis of static game under decentralized decision-making}

Figure 5 shows that the greater the effort coefficient of the supplier, the higher the supplier's effort level, and the less profit sharing of the e-commerce platform; the higher the cost coefficient of the supplier, the lower the supplier's effort level, and the more shares the e-commerce platform have. The greater the risk coefficient of the suppliers, the more they dislike the risks, and the more the e-commerce platform is divided. 

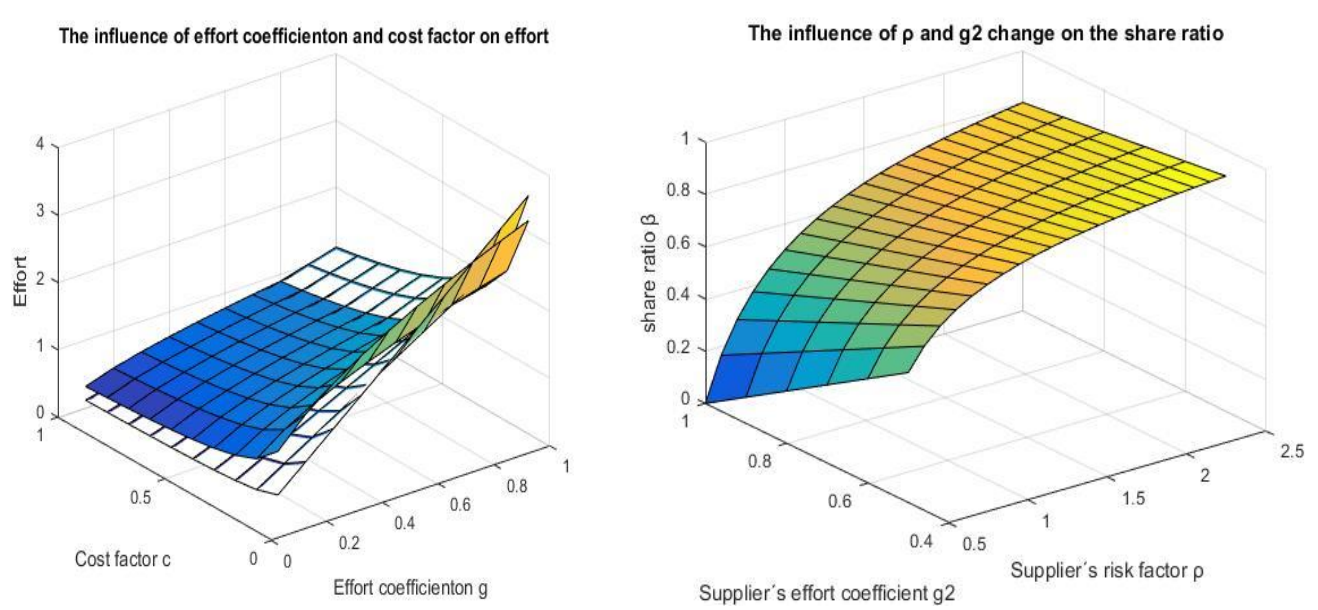

Figure 5. Influence of supplier's risk factor, cost factor, and effort coefficient on effort and share ratio

At this time, the greater the risk coefficient of the supplier and the variance of the random variables, the greater the risk and the lesser the profit of the supplier and the whole supply chain, in addition, the profit sharing of the e-commerce platform is greater.
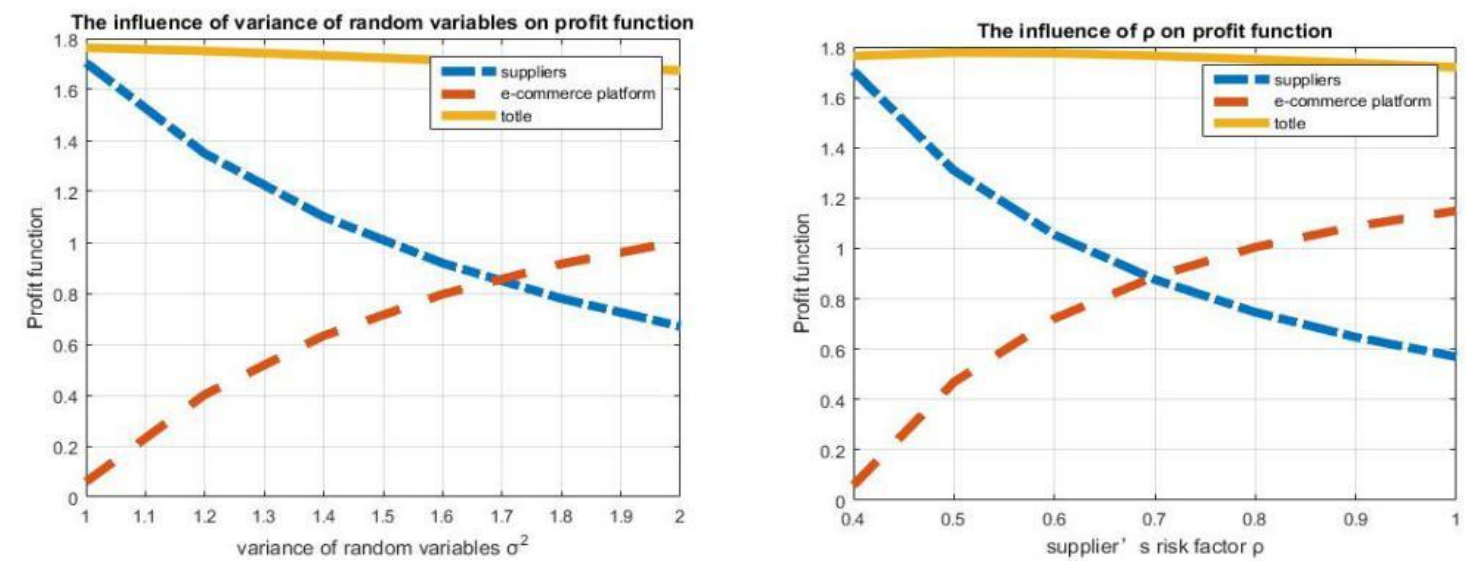

Figure 6. Influence of supplier's risk coefficient and the variance of random variables on profit function

\section{Conclusion}

Divided into three cases, under centralized decision-making, both parties cooperate without considering profit distribution and incentives, and both parties can make the best effort in information sharing to maximize the overall profit. In this case, the level of effort and profit depend on the cost paid and the benefit brought by the effort. However, cooperation between the two parties is often rare, and most cases are decisions made by both parties in a decentralized manner.

In real life, small and medium-sized suppliers tend to avoid risks and have to pay a certain risk cost to participate in information sharing. At this time, e-commerce platforms, as the leader, can adopt a dynamic game strategy and formulate profit distribution rules to motivate suppliers to participate. E-commerce platforms can reduce profit sharing and motivate suppliers to contribute more effort. The more the suppliers work, the higher the degree of information sharing and the more output there would be, so e-commerce platforms can still obtain profits.

On the contrary, strong tourism suppliers tend to have risk preference. They believe that the risk cost is less than the revenue. In this case, e-commerce platforms can adopt a static game strategy, in which both parties make decisions at the same time. E-commerce platforms need to enable the suppliers to participate 
in information sharing and obtain more profits than the original while ensuring the maximum profit for themselves. Compared with the master-slave game, e-commerce platforms need to make more effort for this type of suppliers to increase the level of information sharing efforts in order to achieve greater profits. To sum up, this study provides decision-making methods for tourism supply chains with e-commerce platforms as the core under different circumstances, determines the appropriate degree of information sharing efforts and information sharing costs, as well as achieves the goal of maximizing supply chain profits.

\section{Disclosure statement}

The authors declare that there is no conflict of interest.

\section{References}

[1] Huang M, Shu B, 2015, Research on the Construction of a New Tourism Supply Chain with Online Tourism Service Providers as the Core. Hubei Social Sciences, 04: 83-88.

[2] Zheng S, Fang F, 2014, Study on the Construction of Virtual Cluster Tourism Supply Chain Model. Journal of Tourism, 02: 46-54.

[3] Abou-Shouk M, Lim WM, Megicks P, 2011, Internet Adoption by Travel Agents: A Case of Egypt. Tourism Management, 32(2): 297-306.

[4] Daugherty T, Biocca F, 2005, Experiential E-Commerce: A Summary of Research Investigating the Impact of Virtual Experience on Consumer Learning. Online Consumer Psychology: Understanding and Influencing Consumer Behavior in the Virtual World, 2005(4): 457-489.

[5] Romero I, Tejada P, 2011, A Multi-Level Approach to the Study of Production Chains in the Tourism Sector. Tourism Management, 32(2): 297-306.

[6] Ji Y, 2017, Online Travel Service Supply Chain Coordination Research. Tianjin University.

[7] Xu H, Qi Q, Wang K, et al., 2013, Research on the Construction of Tourism Supply Chain Based on Network Environment. Business Research, 2013(3): 205-211.

[8] Danuta S, 2017, Tourism Supply Chain - Overview of Selected Literature. Procedia Engineering, 182(1): 125-136.

[9] Huang C, 2018, Assessing the Performance of Tourism Supply Chains by Using the Hybrid Network Data Envelopment Analysis Model. Tourism Management, 65(1): 303-316.

[10] Pu XJ, Du X, 2016, "Hotel +OTA" Double Channel Supply Chain Sales Strategy and Coordination Mechanism Research. Journal of Jiangnan University (Humanities and Social Sciences Edition), 15(01): 112-118.

[11] Long Y, Shi P, 2017, Pricing Strategies of Tour Operator and Travel Agency Based on Cooperation to Achieve O2O Model. Tourism Management, 2017: 62. 